\title{
Differential Shedding and Antibody Kinetics of Zika and Chikungunya Viruses, Brazil
}

\author{
Fernando A. Bozza, ${ }^{1}$ Andres Moreira-Soto, ${ }^{1}$ \\ Alexandra Rockstroh, Carlo Fischer, \\ Alessandra D. Nascimento, Andrea S. Calheiros, \\ Christian Drosten, Patrícia T. Bozza, \\ Thiago Moreno L. Souza, \\ Sebastian Ulbert, Jan Felix Drexler
}

In seroconversion panels obtained from patients from Brazil, diagnostic testing for Zika virus infection was improved by combining multiple antibody isotypes, techniques, and antigens, but sensitivity remained suboptimal. In contrast, chikungunya virus diagnostic testing was unambiguous. Recurrent recent arbovirus infections suggested by serologic data and unspecific symptoms highlight the need for exhaustive virologic testing.

$\mathrm{I}^{1}$ 2013, Zika virus and chikungunya virus (CHIKV) emerged in Latin America $(1,2)$. Their overlapping symptoms challenge accurate diagnosis on the basis of clinical manifestations (3). Direct Zika virus and CHIKV detection is limited to the acute phase of infection (4). Serologic detection of Zika virus-specific antibodies is hampered by low specificity and sensitivity of tests because of immune responses elicited by prior infection with other endemic flaviviruses (e.g., dengue virus [DENV]) (5,6). In addition, lack of adequate specimens limits studies evaluating the performance of diagnostic tests in tropical areas $(7,8)$. To evaluate these challenges, we analyzed virus shedding and antibody responses over time in patients in Brazil sampled during the 2016 Zika virus and CHIKV outbreaks.

Author affiliations: National Institute of Infectious Diseases Evandro Chagas, Oswaldo Cruz Foundation (FIOCRUZ), Rio de Janeiro, Brazil (F.A. Bozza, A.D. Nascimento); D'Or Institute for Research and Education, Rio de Janeiro (F.A. Bozza,

A.D. Nascimento); Charité-Universitätsmedizin Berlin, corporate member of Freie Universität Berlin, Humboldt-Universität zu Berlin, and Berlin Institute of Health, Institute of Virology, Berlin, Germany (A. Moreira-Soto, C. Fischer, C. Drosten, J.F. Drexler); Fraunhofer Institute for Cell Therapy and Immunology, Leipzig, Germany (A. Rockstroh, S. Ulbert); Instituto Oswaldo Cruz, Rio de Janeiro (A.S. Calheiros, P.T. Bozza); German Centre for Infection Research, Berlin (C. Drosten, J.F. Drexler); Center for Technological Development in Health, Rio de Janeiro (T.M.L. Souza) Martsinovsky Institute of Medical Parasitology, Tropical and VectorBorne Diseases, Sechenov University, Moscow, Russia (J.F. Drexler)

DOI: https://doi.org/10.3201/eid2502.180166

\section{The Study}

We prospectively sampled patients in 4 time points up to 90 days post-symptom onset (dpo) (Table 1; Figure 1, panel A; Appendix, https://wwwnc.cdc.gov/EID/article/25/2/180166-App1.pdf). The cohort comprised 15 patients with acute Zika virus infection ( 5 male, 10 female; median age 39.0 years [interquartile range $31.0-44.0$ years]) and 18 patients with acute CHIKV infection (10 male, 8 female; median age 39.0 years [interquartile range $31.0-57.3$ years]), determined by detection of viral RNA in blood or urine 1-9 dpo (Appendix Figures 1,2). All Zika virus belonged to the Asian lineage (2), and all CHIKV to the East/Central/South African lineage, according to envelope-based typing.

At enrollment, Zika virus patients most frequently reported fever, rash, and arthralgia ( $80 \%$ each), and CHIKV patients most frequently reported arthralgia (100\%), fever $(89 \%)$, and myalgia (89\%) (Table 2). No co-infection with Zika virus, CHIKV, or DENV was detected by real-time reverse transcription PCR (rRT-PCR). However, serologic analyses found that 4 (27\%) Zika virus-infected patients also had CHIKV IgM at enrollment, and 1 (7\%) had DENV IgM (Appendix Table 1, Figure 3). Similarly, 3 (17\%) CHIKV-infected patients had Zika virus IgM, and 4 (22\%) CHIKV-infected patients had DENV IgM at enrollment (Appendix Figure 4). We cannot exclude the possibility of cross-reactivity between Zika virus-specific and DENVspecific antibodies because 2 CHIKV patients simultaneously showed Zika virus and DENV IgM in an envelopebased ELISA (Appendix Table 2). Seventy-nine percent of Zika virus and $83 \%$ of CHIKV patients showed serologic evidence for past DENV infection at enrollment (Appendix Figures 1,2). Thus, recent infections with heterologous arboviruses might bias attributing infection-specific symptoms for Zika virus and CHIKV.

Consistent with previous studies (4,9), Zika virus loads in serum and urine were low up to 9 dpo $\left(\approx 10^{4}\right.$ RNA copies/mL) (Figure 1, panel B), whereas CHIKV loads were $\approx 100$-fold higher $\left(\approx 10^{6} \mathrm{RNA}\right.$ copies $/ \mathrm{mL}$ ) (Figure 1, panel C). However, unlike with Zika virus, CHIKV loads decreased significantly ( $<<0.001$ by $t$ test) from 5 dpo onward, and viral loads in urine were consistently low (Figure 1, panels D, E).

Next, to assess the antibody kinetics of Zika virus and CHIKV, we measured antibody responses over time

${ }^{1}$ These authors contributed equally to this article. 
Table 1. Sampling details for retrospective study of differential shedding and antibody kinetics of Zika virus and CHIKV, Brazil, 2016*

\begin{tabular}{|c|c|c|c|}
\hline Sample no. & $\begin{array}{c}\text { Virus } \\
\text { detected }\end{array}$ & $\begin{array}{c}\text { Days from } \\
\text { symptom onset } \\
\text { to sampling }\end{array}$ & $\begin{array}{c}\text { Collection date } \\
\text { of acute-phase } \\
\text { samples }\end{array}$ \\
\hline DQ005 & Zika virus & 2 & Mar 14 \\
\hline DQ028 & Zika virus & 1 & Mar 21 \\
\hline DQ042 & Zika virus & 3 & Mar 23 \\
\hline DQ47 & Zika virus & 2 & Mar 28 \\
\hline DQ049 & Zika virus & 1 & Mar 28 \\
\hline DQ058 & Zika virus & 4 & Mar 30 \\
\hline DQ60 & Zika virus & 2 & Mar 30 \\
\hline DQ62 & Zika virus & 3 & Mar 30 \\
\hline DQ75 & Zika virus & 3 & Apr 4 \\
\hline DQ77 & Zika virus & 5 & Apr 5 \\
\hline DQ108 & Zika virus & 2 & Apr 13 \\
\hline DQ116 & Zika virus & 2 & Apr 14 \\
\hline DQ125 & Zika virus & 3 & Apr 18 \\
\hline DQ131 & Zika virus & 5 & Apr 18 \\
\hline DQ246 & Zika virus & 5 & Jun 24 \\
\hline DQ030 & CHIKV & 3 & Mar 21 \\
\hline DQ045 & CHIKV & 5 & Mar 24 \\
\hline DQ054 & CHIKV & 2 & Mar 30 \\
\hline DQ056 & CHIKV & 2 & Mar 30 \\
\hline DQ057 & CHIKV & 3 & Mar 30 \\
\hline DQ061 & CHIKV & 2 & Mar 30 \\
\hline DQ071 & CHIKV & 4 & Apr 4 \\
\hline DQ074 & CHIKV & 1 & Apr 4 \\
\hline DQ079 & CHIKV & 3 & Apr 5 \\
\hline DQ083 & CHIKV & 3 & Apr 6 \\
\hline DQ085 & CHIKV & 4 & Apr 7 \\
\hline DQ097 & CHIKV & 3 & Apr 11 \\
\hline DQ113 & CHIKV & 5 & Apr 13 \\
\hline DQ144 & CHIKV & 4 & Apr 25 \\
\hline DQ170 & CHIKV & 2 & May 3 \\
\hline DQ195 & CHIKV & 2 & May 11 \\
\hline DQ210 & CHIKV & 2 & May 16 \\
\hline DQ220 & CHIKV & 4 & May 17 \\
\hline \multicolumn{4}{|c|}{${ }^{*} \mathrm{CHIKV}$, chikungunya virus. } \\
\hline
\end{tabular}

by commercial and in-house serologic tests. In a widely used nonstructural (NS) protein 1 antigen-based ELISA, Zika virus IgM seroconversion was low (33\% [5/15]), whereas CHIKV IgM seroconversion was $100 \%$ using an envelope-based ELISA $(p<0.0001$ by Fisher exact test) (Figure 2, panel A; Appendix Tables 1,2). Use of an in-house envelope-based ELISA increased the Zika virus $\operatorname{IgM}$ detection rate to $50 \%(7 / 14)$, and use of a commercially available $\mu$-capture ELISA increased it to $43 \%(6 / 14)$ (Figure 2, panel A). Despite differential sensitivity, concordant results from different assays suggest comparable specificity of IgM detection (Appendix Table 1). The use of NS1-based IgA as a marker of acute infection increased the detection rate to $53 \%(8 / 15)$ over that of the NS1-based IgM ELISA. All IgM-positive patients also showed IgA, which increased during acute and subacute phases of infection and decreased during convalescence (Figure 2, panel B; Appendix Figure 3). This finding supports the usability of IgA-based serologic methods as an alternative or additional marker to IgM-based methods to detect acute Zika virus infection.
The detection rate increased 2-fold when we used NS1based IgA from when we used NS1-based IgM 5-9 dpo, suggesting that IgA could be used at later stages of infection (Appendix Figures 1, 5). Our findings indicate that serologic detection of acute Zika virus infection can be improved $\approx 2$-fold by use of different antibody classes and antigens but remains poorly sensitive in flavivirusendemic areas.

All Zika virus-infected patients showed IgG responses across the 4 time points in $\geq 1$ assay (Figure 2, panels C, D). Plaque reduction neutralization tests (PRNTs) were negative for 2 of 14 rRT-PCR-confirmed Zika virus cases detected by NS1-based IgG ELISA. Without rRT-PCR confirmation, these cases would have been classified false positive (Appendix Table 1). This observation might be explained by differential sensitivity of PRNT and ELISA (10) or false-positive results of the Zika virus NS1-based ELISA in secondary flavivirus infections (6). Similarly, the antibody kinetics of Zika virus NS1-based IgG, envelopebased IgG, and PRNT suggested either relatively early IgG seroconversion or cross-reactivity during acute stages of infection resulting from unspecific immune responses against other flaviviruses (11) (Figure 2, panel D). In contrast, CHIKV IgG seroconversion occurred at later stages (Figure 2, panel D; Appendix Figure 5), possibly associated with strong and long-lasting CHIKV-specific IgM responses (Appendix Figure 4).

\section{Conclusions}

We provide pivotal data on Zika virus and CHIKV diagnostic challenges in a Latin American setting. Limitations of our study include the relatively small number of patients, sampling at heterogeneous dpo and heterogeneous numbers of samples per dpo, and lack of acutely DENVinfected patients to assess test specificity. The strengths of our study include rRT-PCR-confirmed infections, waiving the need to define serologic assays prone to crossreactivity as standards, sampling during Zika virus and CHIKV outbreaks $(1,2)$, sequential sampling of patients up to $90 \mathrm{dpo}$, use of multiple antigens and immunoglobulin classes, and the combination of molecular and serologic testing methods.

Our data suggest reliable diagnostic testing for acute CHIKV infections by IgM detection from 5 dpo onward. This finding might enable waiving labor-intense and costly molecular protocols in many patients, minimizing costs for public health systems and cohort studies investigating arbovirus pathogenesis. However, reliability of CHIKV serologic diagnostic tests must be reevaluated for co-circulating genotypes (12) and for the antigenically related Mayaro virus (13) if it emerges in Latin America.

The difficulties of adequately diagnosing Zika virus infections in areas to which it is endemic have major 


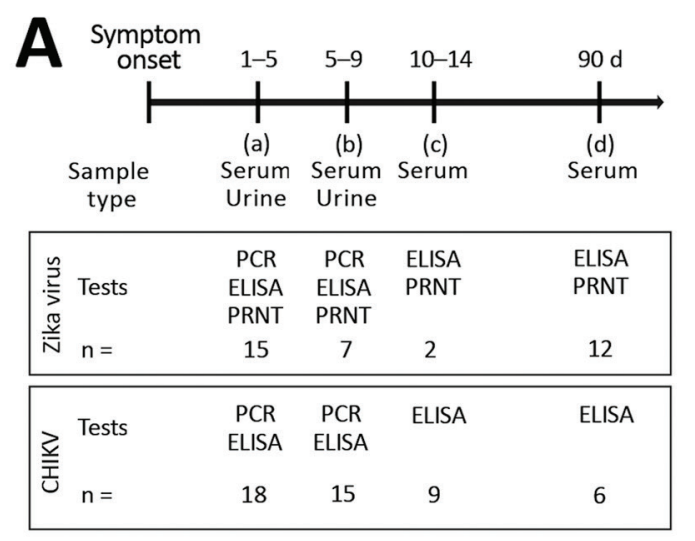

D
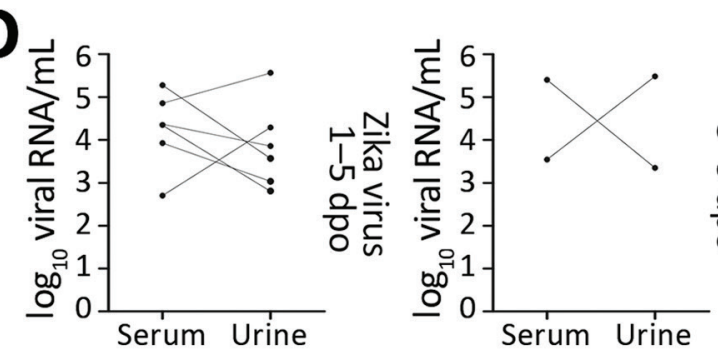
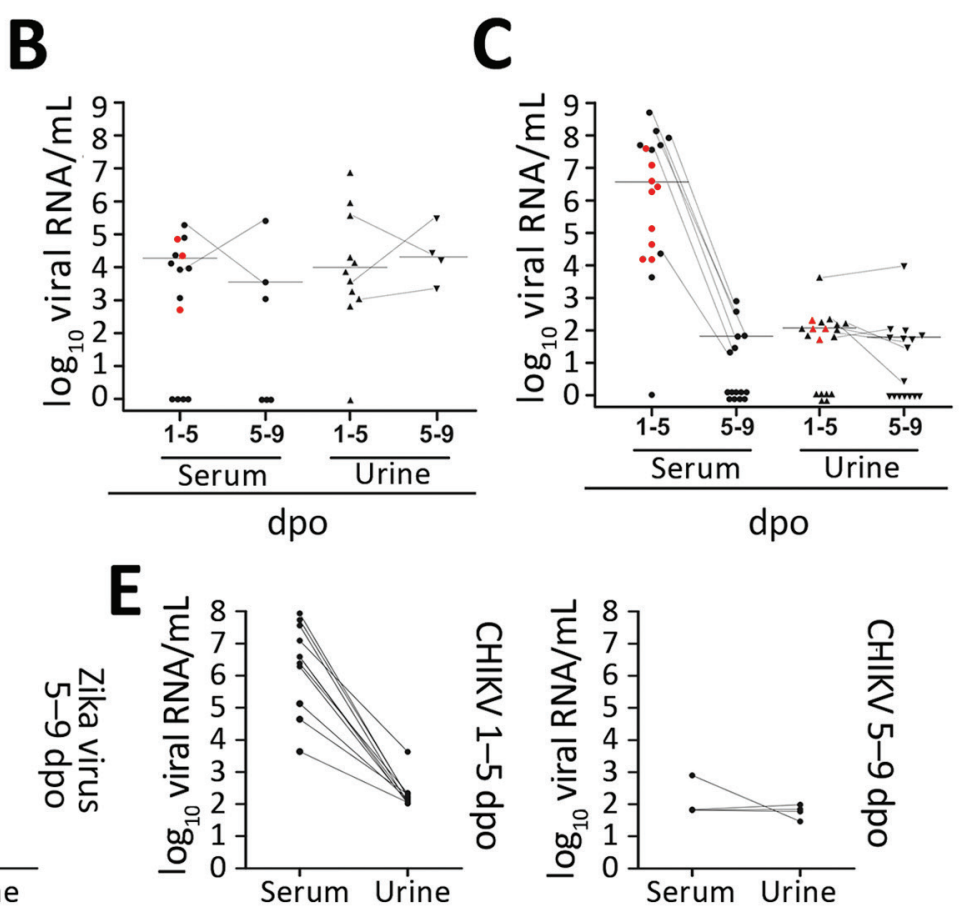

Figure 1. Overview of diagnostic testing and shedding dynamics for Zika virus and CHIKV among patients in Brazil, 2016. A) Timeline of sampling and number of samples for each test. B, C) Zika virus (B) and CHIKV (C) loads in different body fluids at 1-5 and 5-9 dpo. Black dots indicate single samples. Red dots indicate samples taken 1-5 dpo that were negative 5-9 dpo. Dotted lines indicate paired samples that were positive at both time points. Bold line indicates the median. D, E) Viral loads of Zika virus (D) and CHIKV (E) in paired urine and serum samples from individual patients, 1-5 and 5-9 dpo. Data were analyzed using GraphPad Prism 5 (GraphPad Software, Inc., https://www.graphpad.com). CHIKV, chikungunya virus; dpo, days post-symptom onset; PRNT, plaque reduction neutralization test.

implications for public health. Reliable testing for flaviviruses in such areas will be key for epidemiologic studies on Zika virus and assessments of the safety of flavivirus vaccination programs, as illustrated by more severe dengue infections in DENV-seronegative individuals who received a live attenuated dengue vaccine (14).

For pregnant women and couples intending pregnancy, accurate diagnosis of acute or past Zika virus infection is crucial. The steep increase in requests for abortion in Latin America illustrates the effect of the Zika virus outbreak on reproductive medicine (15).

Table 2. Symptoms of Zika virus and CHIKV reported by patients at enrollment 1-5 days after symptom onset, Brazil, 2016*

\begin{tabular}{lcc}
\hline Symptom & $\begin{array}{c}\text { Zika virus, no. (\%), } \\
\mathrm{n}=15\end{array}$ & $\begin{array}{c}\text { CHIKV, no. }(\%), \\
\mathrm{n}=18\end{array}$ \\
\hline Rash & $12(80)$ & $9(50)$ \\
Fever & $12(80)$ & $16(89)$ \\
Arthralgia & $12(80)$ & $18(100)$ \\
Myalgia & $9(60)$ & $16(89)$ \\
Cephalea & $8(53)$ & $12(67)$ \\
Retro-orbital pain & $5(33)$ & $8(44)$ \\
Edema & $4(27)$ & $3(17)$ \\
Nausea, vomiting & $3(20)$ & $6(33)$ \\
Conjunctivitis & $2(13)$ & $5(28)$ \\
\hline${ }^{*}$ CHIKV, chikungunya virus & &
\end{tabular}

${ }^{*} \mathrm{CHIKV}$, chikungunya virus.
Our results highlight that definite exclusion of acute Zika virus infections is challenging in a considerable proportion of patients. However, although limited by a small number of samples, our data highlight the attainability of more accurate Zika virus diagnostic testing by combining molecular and serologic tests using different antibody classes, antigens, and methods and by monitoring an increase of IgG titers in follow-up serum samples. Our data will help clinicians and health authorities build reliable diagnostic algorithms for Zika virus and CHIKV and highlight that exhaustive testing of arboviral infections is required for attributing frequencies of infection-specific symptoms.

\section{Acknowledgments}

We thank Ignacio Postigo-Hidalgo, Jens Miguel Warnecke, and Thiago Carvalho for technical assistance.

This work was supported by the European Union's Horizon 2020 Research and Innovation Programme through the ZIKAlliance project (grant agreement no. 734548), the German Centre for Infection Research through the ZIKApath project, by the Conselho Nacional de Desenvolvimento e Pesquisa (CNPq) and Fundação de Amparo à Pesquisa do Estado do Rio de Janeiro (FAPERJ). 
Figure 2. Zika virus and CHIKV antibody dynamics among samples from patients in Brazil, 2016. A) Percentage seroconversion for markers of acute infection with Zika virus and CHIKV (IgM NS1-based Zika virus ELISA, IgM envelopebased Zika virus ELISA, IgM $\mu$-capture Zika virus ELISA, IgA NS1-based Zika virus ELISA, IgM CHIKV ELISA) at any time point. B) Median ELISA ratios for Zika virus and CHIKV IgM and IgA over time. C) Percentage seroconversion for markers of convalescence after Zika virus and CHIKV infection (IgG NS1based Zika virus ELISA and IgG envelope-based Zika virus ELISA, Zika virus PRNT $_{50}$, IgG CHIKV ELISA) at any time point. D) Median ELISA ratios for Zika virus and CHIKV IgG over time. Numbers of specimens per time point are shown in Figure 1. Dashed lines indicate signal-tocutoff ratios of $\geq 1.1$ considered positive for all ELISAs except $\mu$-capture ELISA, for which the dashed line indicates a signalto-cutoff ratio of $\geq 10$, considered

A

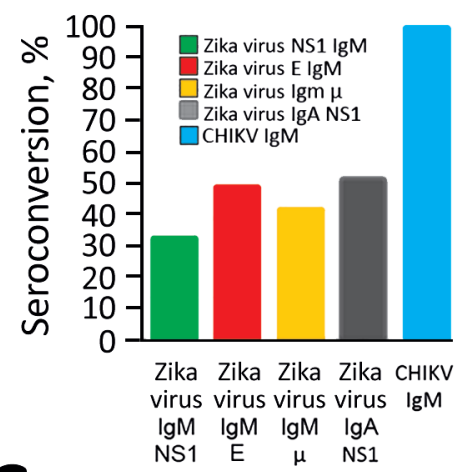

$C$

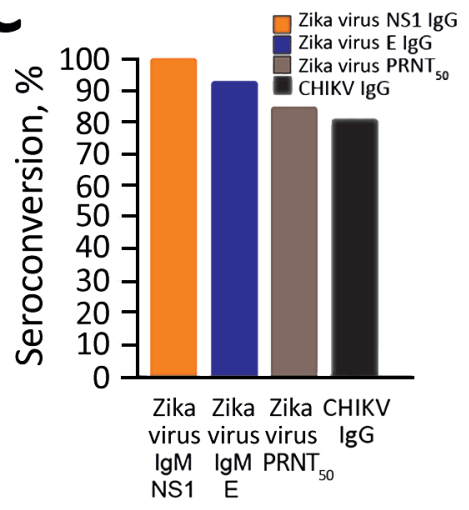

B

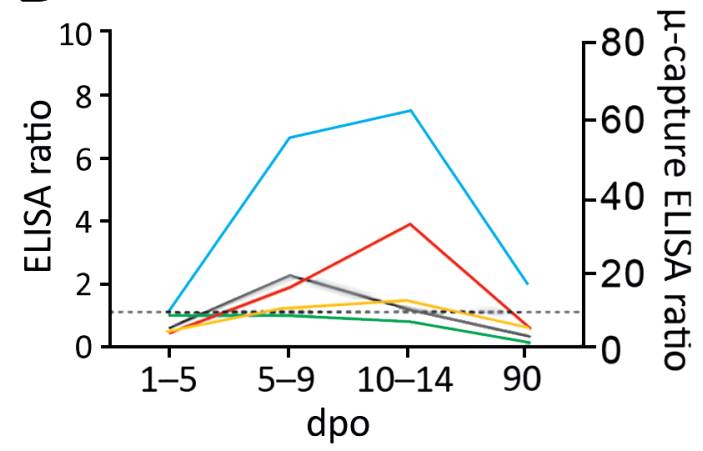

D

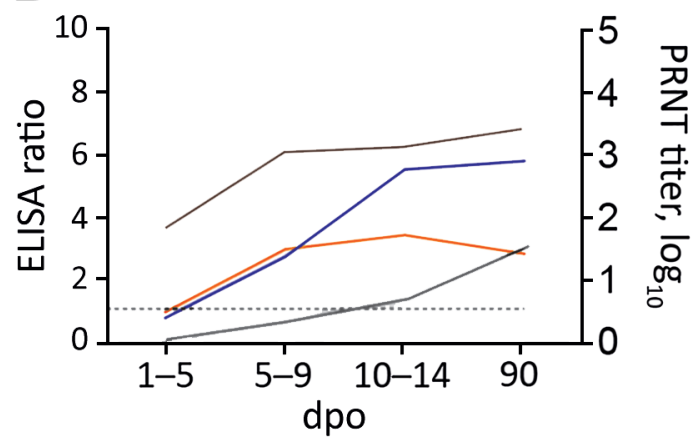
positive by the manufacturer. See Appendix Figure 5 (https://wwwnc.cdc.gov/EID/article/25/2/18-0166-App1.pdf) for the percentage de novo seroconversion of Zika virus and CHIKV in different assays per time point. CHIKV, chikungunya virus; dpo, days postsymptom onset; E, envelope; NS, nonstructural protein; PRNT, plaque reduction neutralization test.

\section{About the Author}

Dr. F.A. Bozza is a senior scientist and head of the Laboratorio de Medicina Intensiva, Fundação Oswaldo Cruz Rio de Janeiro. His research focuses on the host immune response and metabolic adaptation to severe infections.

\section{References}

1. Nunes MR, Faria NR, de Vasconcelos JM, Golding N, Kraemer MU, de Oliveira LF, et al. Emergence and potential for spread of chikungunya virus in Brazil. BMC Med. 2015;13:102. http://dx.doi.org/10.1186/s12916-015-0348-x

2. Metsky HC, Matranga CB, Wohl S, Schaffner SF, Freije CA, Winnicki SM, et al. Zika virus evolution and spread in the Americas. Nature. 2017;546:411-5. http://dx.doi.org/10.1038/ nature 22402

3. Musso D, Gubler DJ. Zika Virus. Clin Microbiol Rev. 2016;29:487-524. http://dx.doi.org/10.1128/CMR.00072-15

4. Corman VM, Rasche A, Baronti C, Aldabbagh S, Cadar D, Reusken CB, et al. Assay optimization for molecular detection of Zika virus. Bull World Health Organ. 2016;94:880-92. http://dx.doi.org/10.2471/BLT.16.175950

5. Netto EM, Moreira-Soto A, Pedroso C, Höser C, Funk S, Kucharski AJ, et al. High Zika virus seroprevalence in Salvador, northeastern Brazil limits the potential for further outbreaks. MBio. 2017;8:01390-17. http://dx.doi.org/10.1128/mBio.01390-17
6. Felix AC, Souza NCS, Figueiredo WM, Costa AA, Inenami M, da Silva RMG, et al. Cross reactivity of commercial anti-dengue immunoassays in patients with acute Zika virus infection. J Med Virol. 2017;89:1477-9. http://dx.doi.org/10.1002/jmv.24789

7. Steinhagen K, Probst C, Radzimski C, Schmidt-Chanasit J, Emmerich P, van Esbroeck M, et al. Serodiagnosis of Zika virus (ZIKV) infections by a novel NS1-based ELISA devoid of crossreactivity with dengue virus antibodies: a multicohort study of assay performance, 2015 to 2016. Euro Surveill. 2016;21:30426. http://dx.doi.org/10.2807/1560-7917.ES.2016.21.50.30426

8. L'Huillier AG, Hamid-Allie A, Kristjanson E, Papageorgiou L, Hung S, Wong CF, et al. Evaluation of Euroimmun anti-Zika virus IgM and IgG enzyme-linked immunosorbent assays for Zika virus serologic testing. J Clin Microbiol. 2017;55:2462-71. http://dx.doi.org/10.1128/JCM.00442-17

9. Jain J, Nayak K, Tanwar N, Gaind R, Gupta B, Shastri JS, et al. Clinical, serological, and virological analysis of 572 chikungunya patients from 2010 to 2013 in India. Clin Infect Dis. 2017;65:13340. http://dx.doi.org/10.1093/cid/cix283

10. Lindsey NP, Staples JE, Powell K, Rabe IB, Fischer M, Powers AM, et al. Ability to serologically confirm recent Zika virus infection in areas with varying past incidence of dengue virus infection in the United States and U.S. territories in 2016. J Clin Microbiol. 2017;56:e01115-7. http://dx.doi.org/10.1128/ JCM.01115-17

11. Priyamvada L, Quicke KM, Hudson WH, Onlamoon N, Sewatanon J, Edupuganti S, et al. Human antibody responses after dengue virus infection are highly cross-reactive to Zika virus. Proc 
Natl Acad Sci U S A. 2016;113:7852-7. http://dx.doi.org/10.1073/ pnas. 1607931113

12. Weaver SC, Lecuit M. Chikungunya virus and the global spread of a mosquito-borne disease. N Engl J Med. 2015;372:1231-9. http://dx.doi.org/10.1056/NEJMra1406035

13. Hassing RJ, Leparc-Goffart I, Tolou H, van Doornum G, van Genderen PJ. Cross-reactivity of antibodies to viruses belonging to the Semliki forest serocomplex. Euro Surveill. 2010;15:19588.

14. World Health Organization. Updated questions and answers related to the dengue vaccine Dengvaxia $\AA$ and its use [cited 2018
Jan 30]. http://www.who.int/immunization/diseases/dengue/ $\mathrm{q}$ and a dengue vaccine dengvaxia use/en/

15. Aiken ARA, Scott JG, Gomperts R, Trussell J, Worrell M, Aiken CE. Requests for abortion in Latin America related to concern about Zika virus exposure. N Engl J Med. 2016;375: 396-8. http://dx.doi.org/10.1056/NEJMc1605389

Address for correspondence: Jan Felix Drexler, Helmut-Ruska-Haus, Institute of Virology, Campus Charité Mitte, Charitéplatz 1, 10098 Berlin, Germany; email: felix.drexler@charite.de

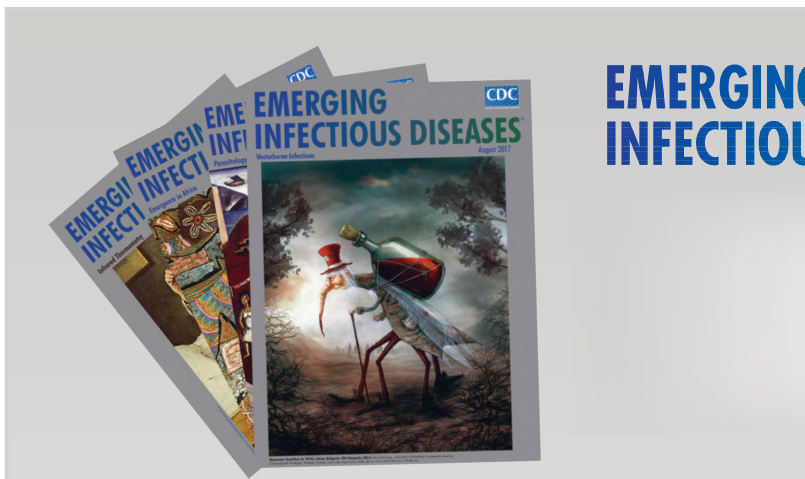

- Added Value of Next-Generation Sequencing for Multilocus Sequence Typing Analysis of a Pneumocystis jirovecii Pneumonia Outbreak

- Bartonella quintana, an Unrecognized Cause of Infective Endocarditis in Children in Ethiopia

- Characteristics of Dysphagia in Infants with Microcephaly Caused by Congenital Zika Virus Infection, Brazil, 2015

- Zika Virus Infection in Patient with No Known Risk Factors, Utah, USA, 2016

- Acute Febrile Illness and Complications Due to Murine Typhus, Texas, USA

- High Infection Rates for Adult Macaques after Intravaginal or Intrarectal Inoculation with Zika Virus

- Lyme Borreliosis in Finland, 1995-2014

- Characterization of Fitzroy River Virus and Serologic Evidence of Human and Animal Infection

- Genomic Characterization of Recrudescent Plasmodium malariae after Treatment with Artemether/Lumefantrine

- Molecular Characterization of Corynebacterium diphtheriae Outbreak Isolates, South Africa, March-June 2015

- Clinical Laboratory Values as Early Indicators of Ebola Virus Infection in Nonhuman Primates

- Maguari Virus Associated with Human Disease

- Human Infection with Highly Pathogenic Avian Influenza $\mathrm{A}(\mathrm{H} 7 \mathrm{~N} 9)$ Virus, China

- Genesis of Influenza A(H5N8) Viruses

\section{August 2017}

\section{Vectorborne Infections}

- Human Metapneumovirus and Other Respiratory Viral Infections during Pregnancy and Birth, Nepal

- Global Spread of Norovirus Gll.17 Kawasaki 308, 2014-2016

- Preliminary Epidemiology of Human Infections with Highly Pathogenic Avian Influenza A(H7N9) Virus, China, 2017

- Real-Time Evolution of Zika Virus Disease Outbreak, Roatán, Honduras

- Clonal Expansion of New Penicillin-Resistant Clade of Neisseria meningitidis Serogroup W Clonal Complex 11, Australia

- Density-Dependent Prevalence of Francisella tularensis in Fluctuating Vole Populations, Northwestern Spain

- Occupational Exposures to Ebola Virus in Ebola Treatment Center, Conakry, Guinea

- West Nile Virus Outbreak in Houston and Harris County, Texas, USA, 2014

- Serologic Evidence of Scrub Typhus in the Peruvian Amazon

- Serologic Evidence of Powassan Virus Infection in Patients with Suspected Lyme Disease

- Influenza D Virus in Animal Species in Guangdong Province, Southern China

- Seroprevalence of Baylisascaris procyonis Infection among Humans, Santa Barbara County, California, USA, 2014-2016

- Opiate Injection-Associated Skin, Soft Tissue, and Vascular Infections, England, UK, 1997-2016 